\title{
Physical activity and obesity: is there a difference in association between the Asian- and African- Surinamese adult population?
}

Running head: Physical activity and obesity: ethnic differences?

Se-Sergio M. Baldew ${ }^{1,2}$, Frederieke S. Diemer ${ }^{3,4}$, Veronique Cornelissen², Glenn P. Oehlers ${ }^{3}$, Lizzy M. Brewster ${ }^{4}$, Jerry R. Toelsie ${ }^{5}$ and Luc Vanhees ${ }^{2}$

${ }^{1}$ Department of Physical Therapy, Faculty of Medical Sciences, Anton de Kom University of Suriname, Paramaribo, Suriname

${ }^{2}$ Department of Rehabilitation Sciences, Research Center for Cardiovascular Rehabilitation, Faculty of Kinesiology and Rehabilitation Sciences, KULeuven, Leuven, Belgium

${ }^{3}$ Department of Cardiology, Academic Hospital of Paramaribo, Suriname

${ }^{4}$ Department of Vascular, Internal and Social Medicine, Academic Medical Center, Amsterdam, the Netherlands

${ }^{5}$ Department of Physiology, Faculty of Medical Sciences, Anton de Kom University of Suriname, Paramaribo, Suriname.

Se-Sergio M. Baldew : sergio.baldew@uvs.edu (corresponding author)

Frederieke S. Diemer: f.s.diemer@amc.uva.nl.

Veronique Cornelissen: veronique.cornelissen@kuleuven.be

Glenn P. Oehlers: gpoelhers@ gmail.com

Lizzy M. Brewster: 1.m.brewster@amc.nl

Jerry R. Toelsie: j.toelsie@uvs.edu

Luc Vanhees: luc.vanhees@ kuleuven.be 
Physical activity and obesity: ethnic differences?

\begin{abstract}
Objective: The role of different physical activity (PA) characteristics, i.e. domain, duration and intensity in obesity prevention still requires investigation. Furthermore, ethnicity can modify the effect of PA on body composition. Therefore, we aim to describe the association between obesity and PA characteristics across the Asian- and African-Surinamese population, living in the capital of Suriname.
\end{abstract}

Design: Between February 2013 and July 2015, we included 1157 healthy subjects, 18 -70 years, from the Healthy Life in Suriname (HELISUR) study. We measured height, weight, hip and waist circumference and defined general and central obesity according to World Health Organization (WHO) recommendations. The International Physical Activity Questionnaire was used to assess PA and to calculate the duration (minutes/week) and the total volume (METsminutes/week) of activity. Ethnicity was self-reported.

Results: Out of 1157 participants we included 1079 (42.6\% Asian-Surinamese, 40.1\% AfricanSurinamese and $17.3 \%$ of other ethnicity), mean age $42.6 \pm 13.6$ years for analysis. Obesity prevalence ratio (PR) was significantly lower in participants meeting WHO PA recommendations $[\mathrm{PR}=0.81(0.68-0.97)]$, especially within the commuting $[\mathrm{PR}=0.66(0.47-$ 0.91)] and leisure time domains $[\mathrm{PR}=0.67(0.47-0.94)]$ compared to participants that did not meet the recommendations. Active minutes/week and total volume of activity were inversely associated with obesity and waist circumference, in the overall $(\mathrm{p}<0.05)$ and in the AfricanSurinamese population $(\mathrm{p}<0.05)$, but not in the Asian-Surinamese population.

Conclusion: Meeting PA recommendations, particularly within the commuting and leisure time domains, is associated with lower obesity prevalence in the total population. Among the AfricanSurinamese population, PA within the leisure time domain, more active minutes/week and 
Physical activity and obesity: ethnic differences?

higher levels of total volume are associated with a lower obesity prevalence. This is not found in the Asian-Surinamese population.

Keywords: Physical activity; Ethnicity; Asian descendants; African descendants; Body composition; Health behavior; 
Physical activity and obesity: ethnic differences?

\section{Introduction}

Over one third of the world's adult population is overweight and at least $13 \%$ is obese [1]. Unfortunately, these numbers are increasing worldwide and at a faster rate in low- and middleincome countries, particularly in urban settings [2]. Besides being the fifth leading cause of death [3], obesity is an important cardiovascular risk factor and has also many adverse effects on other cardiovascular risk factors, including diabetes mellitus type 2, hypertension, and dyslipidemia [4, 5]. Therefore, there is an urgent need for effective treatment strategies.

Physical activity (PA) is a key element in the management of obesity [6] and the American College of Sports Medicine recommends individuals to accumulate at least150 to 250 minutes of moderate to vigorous PA per week with a targeted energy expenditure of $400 \mathrm{kcal}$ per day [7]. However, uncertainties remain with regard to the importance of each of the different PA characteristics [8-12], i.e. domain, duration or intensity, that determines optimal weight control [13-16]. Furthermore, these guidelines are based on data derived mainly from Caucasian populations, even though there is clear evidence that variations exist in body composition and cardiovascular risk factors between other ethnicities and Caucasians [17-20]. Moreover, small studies suggest that the responses to PA interventions might be different across ethnic groups [21, 22], underlining the need for ethnic-specific PA recommendations for weight management.

Suriname is a middle-income country in the north-eastern part of South America, but culturally and economically has more similarities with the Caribbean. Due to historical migrations, the population of Suriname consists predominantly of people from Asian and African descent. This provides a unique opportunity to compare ethnic differences between these groups living within the same area. As such, the Healthy LIfe in SURiname (HELISUR) study was designed to describe the prevalence of cardiovascular risk factors, intermediate endpoints, and 
cardiovascular diseases among the different ethnic groups living within the capital Paramaribo [23]. The current report uses data from the HELISUR project to describe the association between obesity prevalence and PA characteristics (domain, duration and intensity) among the total population and across the Asian- and African-Surinamese population living in Paramaribo.

\section{Methods}

\section{Study population}

A detailed description of the HELISUR study design and participant recruitment has been published previously [23]. In short, between February 2013 and July 2015 a total of 1800 randomly selected non-institutionalized individuals (18 to 70 years) living in the capital Paramaribo were contacted and interviewed at home by trained interviewers. From the recruited participants, 1157subjects were further examined at the academic hospital in Paramaribo. Ethical clearance was obtained from the Ethics Committee of the Ministry of Health in Suriname (Approval nr. VG021-2012) and written informed consent was provided by all participants.

\section{Ethnicity}

Participants were mainly Asian-Surinamese (self-identified South Asian and Indonesian descent); African-Surinamese (self-identified Creoles, that lived in Paramaribo for several generations and Maroons, that migrated from the rural interior area to Paramaribo in the past 1020 years) or other ethnicity (Chinese, Amerindians and Caucasians). In the current report we focused on the Asian and the African-Surinamese group, comprising the four main ethnic groups living in Paramaribo. 
Physical activity and obesity: ethnic differences?

\section{Assessment of PA}

The Dutch version of the International Physical Activity Questionnaire-long form (IPAQ-LF) was used to assess PA. This version has been validated previously [24] and was pre-tested for reliability and face-validity in a small study sample. Participants were asked about their activities within the 1) working, 2) commuting, 3) domestic and garden, and 4) leisure time domain during the last seven days. Activities that were performed as part of paid or unpaid work were classified as activities within the working domain, whereas cycling and/or walking from one place to another were defined as activities within the commuting domain. Chores within and around the house were classified as activities within the domestic and garden domain whereas walking activities and/or activities that were not included within the former domains were classified within the leisure time domain. Within every domain the subjects were asked about the number of days and the amount of time they spend doing these activities and at what intensity. Activities could be performed at moderate or vigorous intensity or at a walking level. Moderate intensity was defined as experiencing a slight increase in heartrate and breathing rate, whereas vigorous intensity was defined as experiencing a significant increase in heartrate and breathing rate. Furthermore, an activity within each domain and intensity had to be performed for at least 10 minutes continuously to be considered valid.

The "Guidelines for data processing and analysis of the IPAQ: short and long form" [25] were used to clean and analyze the data. These guidelines provide information on how to calculate the duration of activity per week (minutes/week) for the domains separately and combined. This is done by multiplying the number of days by the amount of time spend per day being active for each domain and combined. Also, the PA volume was calculated for each participant by multiplying the duration by the assigned metabolic equivalent (MET) value for a specific activity 
within each domain (METs-minutes/week). The guidelines provide these MET values for the intensity of each activity, ranging from 3.0 to 8.0 METs. The total volume of PA is then calculated by combining the calculation of all the domains. Subsequently, participants who met at least one of the following recommendations were categorized as meeting the recommended level of PA that corresponds to the WHO guidelines for PA:

1. Physical activity on at least 3 days of the week at a vigorous intensity for at least 20 minutes/day

2. Physical activity on at least 5 days of the week at moderate intensity or walking for at least 30 minutes/day

3. Physical activity on at least 5 days of the week of any combination of walking, moderate or vigorous intensity activities with a minimum of $600 \mathrm{MET}-\mathrm{minutes} /$ week

4. Physical activity on at least 3 days of the week at a vigorous intensity achieving a minimum of at least $1500 \mathrm{MET}$-minutes/week

5. Physical activity of any combination of walking, moderate or vigorous intensity activity with a minimum of 3000 MET-minutes/week

\section{Assessment of body composition}

During the hospital visit, data on body composition were assessed by a single investigator [23]. Waist circumference (WC) was measured at the midpoint between the lower margin of the least palpable rib and the top of the iliac crest, whereas the hip circumference was measured at the broadest circumference below the waist, using a stretch resistant measuring tape. In order to calculate the body mass index (BMI: $\mathrm{kg} / \mathrm{m}^{2}$ ), height and weight were measured using a stadiometer (SECA) and a scale (SECA 840), respectively. All four measurements were done in duplicate and a third measurement was made if the difference between the first two readings was 
$>1.0 \mathrm{~cm}$ for the circumferences, $>0.5 \mathrm{~cm}$ for height and $>0.5 \mathrm{~kg}$ for weight. Mean values were used in the analysis. For the definition of overweight and obesity, we used the ethnic-specific BMI cut-off values that correspond to a high risk for undesirable health proposed by the WHO. For the African-Surinamese and other ethnicities overweight was defined as BMI $\geq 25 \mathrm{~kg} / \mathrm{m}^{2}$ and obesity as BMI $\geq 30 \mathrm{~kg} / \mathrm{m}^{2}$, whereas for the Asian-Surinamese overweight was defined as BMI $\geq$ $23 \mathrm{~kg} / \mathrm{m}^{2}$ and obesity as BMI $\geq 27.5 \mathrm{~kg} / \mathrm{m}^{2}[26,27]$. Central obesity was defined using the ethnicspecific criteria of the International Diabetes Federation [27, 28]. For the Asian-Surinamese participants, the cut-off values of $\geq 90 \mathrm{~cm}$ for men and $\geq 80 \mathrm{~cm}$ for women were used and for the African-Surinamese and other ethnicity participants the cut-off values of $\geq 94 \mathrm{~cm}$ for men and $\geq$ $80 \mathrm{~cm}$ for women were used [27, 28].

\section{Statistical analysis}

Statistical analysis was performed using SPSS (version 21, SPSS Inc., Chicago, IL). Sensitivity analysis was done to compare the participants that were interviewed with the participants that were interviewed and completed the physical examination. Furthermore, analyses were made for the total population and for each of the two main ethnic groups: Asian-Surinamese and AfricanSurinamese. Continuous variables were tested for normality by visual inspection of the histogram. Descriptive data were reported as means \pm standard deviations, medians (range), or percentages; differences between both ethnic groups were tested with an ANOVA or chi-square test. For the total PA and for the separate characteristics (i.e. domain, duration and intensity), the percentage of participants meeting the recommended PA level were calculated for the total population and for each ethnic group. 
In order to compare the obesity prevalence in participants meeting the recommended PA level and those not meeting the recommended level, we estimated prevalence ratios (PR) with corresponding 95\% confidence intervals $(95 \% \mathrm{CI})$ using Poisson regression models with robust variance [29] and adjusted for sex and age.

The continuous variables duration (minutes/week), mean intensity (METs) and volume (METsminutes/week) were categorized into tertiles. For the total population and for each ethnic group, the obesity PR (95\% CI) within each tertile was calculated using the lowest tertile as the reference group. For BMI and WC, the mean values within each tertile of the duration, intensity and total volume were compared. This was done for the total population and for each ethnic group using an ANOVA test with the significance level set at a two-tailed p-value $<0.05$.

\section{Results}

From the 1157 participants that underwent physical examination, 78 were excluded for the final analysis, either because of missing $(n=19)$ or invalid PA data $(n=7)$, a history of coronary heart disease $(n=22)$ or stroke $(n=30)$, resulting in a sample of 1079 participants [42.6\% AsianSurinamese $(n=460), 40.1 \%$ African-Surinamese $(n=432)$ and17.3\% other ethnicity $(n=187)]$. The other ethnicity group included mainly participants of mixed ethnicity $(n=164)$, but also a small number of Amerindians ( $\mathrm{n}=13)$, Chinese-Surinamese $(\mathrm{n}=2)$, and people of other descent $(\mathrm{n}=8)$ (Figure 1).

Table 1 shows the characteristics of the total study population and the two ethnic groups. The majority of the population was female $(63.9 \%)$ with an equal distribution between both ethnic groups ( $\mathrm{p}=0.52$ ). BMI was significantly higher in individuals of African-Surinamese origin $(\mathrm{p}<0.01)$. However, based on the current definitions for overweight and central obesity, both 
Physical activity and obesity: ethnic differences?

were significantly more prevalent among the Asian-Surinamese population (both $\mathrm{p}<0.01$ ) whereas there was no difference with regard to the prevalence of obesity across both ethnic groups $(\mathrm{p}=0.81)$. Overall, $78.5 \%$ of the participants met the PA recommendations, with the Asian-Surinamese being slightly more active compared to African Surinamese (82.2\% in AsianSurinamese vs. $76.6 \%$ in African-Surinamese, $\mathrm{p}<0.05)$.

For the total sample, the age and sex-adjusted prevalence of obesity was $19 \%$ lower in participants who met the recommendations for $\mathrm{PA}(\mathrm{PR}=0.81$ [95\% CI $0.68-0.91])$, as depicted in Table 2. Focusing on each of the domains of PA, the prevalence of obesity was 33-34\% lower in participants who met the recommended level of PA within the commuting or leisure time domain [for the commuting domain: $\mathrm{PR}=0.66$ (95\% CI 0.47—0.91); for the leisure time domain: $\mathrm{PR}=0.67(95 \% \mathrm{CI} 0.47-0.94)]$. For the work and domestic and garden domain we did not find a significant association. Regarding the ethnic subgroup analysis, obesity was significantly less prevalent among African-Surinamese individuals meeting the PA recommendations within the leisure time domain $[\mathrm{PR}=0.43(0.20-0.91)]$ compared to those that did not. This association could not be observed in the Asian-Surinamese population [PR=0.78 $(0.49-1.24)]$. For the three remaining domains, we did not find a significant association in both the Asian- and the AfricanSurinamese population.

As shown in Table 3, obesity was less prevalent in the overall sample among the participants being active for at least 1141 minutes/week $[\mathrm{PR}=0.79(0.66-0.94)]$ or performing more than 5281METs-minutes/week [PR=0.79 $(0.66-0.95)]$. Similar results could be observed for the subgroup of individuals from African-Surinamese origin, but not in the Asian-Surinamese individuals. No association between PA intensity and obesity prevalence could be established for the total population and for both ethnic groups. 
Finally, as shown in Table 4, the mean BMI and WC decreased with increasing duration, intensity and volume for the overall population. For the African-Surinamese population both the BMI and WC decreased with increasing duration, intensity and volume, whereas for the AsianSurinamese population only the intensity within the highest tertile significantly lowered the BMI, but not the WC, compared to the other tertiles.

As shown in figure 1 , from the 1800 recruited and interviewed participants, 1157 completed the physical examination. Sensitivity analysis showed that there were no differences between participants who were only interviewed and participants who were interviewed and completed the physical examination in sex, prevalence of smoking and meeting the WHO recommendations for PA $(78.5 \%$ vs $81.4 \%$, p>0.05). However, participants who were also physically examined were significantly older $(42.6 \pm 13.6$ vs $35.5 \pm 14.0, p<0.05)$ more often African-Surinamese (51.4\%vs 40.1\%], $p<0.05)$ and less often Asian-Surinamese (31.4\% vs 42.6\%, $p<0.05)$.

\section{Discussion}

The results of this substudy of the HELISUR project show that 1) overweight and central obesity are more prevalent among the Asian-Surinamese compared to the African-Surinamese population living in urban Paramaribo; 2) obesity prevalence is similar in both ethnic groups; 3) meeting the PA recommendations and being active within the commuting and leisure time domain are all inversely associated with obesity prevalence in the total population; 4) prevalence ratios for obesity are lower in individuals spending more time to PA and performing larger volumes both in the total population as well as within the African-Surinamese population; 5) no 
Physical activity and obesity: ethnic differences?

association could be established between PA and obesity prevalence in individuals of Asian origin; 6) body mass index and waist circumference were significantly lower with increasing duration, intensities and volume in the total population and in the African-Surinamese population; 7) only BMI was significantly lower in the Asian-Surinamese population in the highest tertile of intensity.

The results of our study are in line with previous studies showing that meeting the weekly recommended amount of PA, as formulated by the WHO, is inversely associated with obesity $[30,31]$. This was especially true for being active within the commuting and leisure time domain $[9,12]$. However, we could not establish such association for work-related and domestic and garden activities. There are likely various reasons for these different associations across the PA domains and obesity. First, we used self-reported PA data, which is sensitive to social desirability bias, recall bias, and overestimation [32-34]. Foong et al [35] also reported that the adiposity and PA association was stronger for accelerometer-determined PA than questionnairedetermined PA. Therefore, an overestimation could have masked an existing association among the working and domestic and garden domain. For these two domains in particular, subjects find it very difficult to define the exact amount of time and the intensity of the activities [32, 33]. Commuting and leisure time activities are more planned activities allowing the participants to estimate the time and intensity spend in these domains more accurately [34].

We also found a lower prevalence ratio of obesity among individuals of African origin meeting the recommended levels of PA in their leisure time, but not in individuals of Asian origin. Also, more time spend to PA and higher volumes were associated with lower prevalence ratio's in the African-Surinamese but not in the Asian-Surinamese population. These results are in line with Kwon, Wang and Hawkins (2013) who reported an inverse relation between leisure time PA and 
Physical activity and obesity: ethnic differences?

obesity among the African but not among the Asian population[36]. The reason for this finding remains unclear and warrants further research. The use of categorical data leading to statistical power loss might be one of the reasons. However, we hypothesize that the Asian-Surinamese population is more sensitive for social desirable reporting [37], leading to over reporting of PA compared to individuals of African origin. To this end, the use of more objective instruments to quantify PA is necessary.

Among the African-Surinamese population the BMI and WC were consistently lower in the highest tertiles for duration, intensity and volume. Among the Asian-Surinamese only the BMI was significantly lower in the highest intensity tertile compared to the lowest tertile. Even though Lesser et al.(2012) did report that visceral adipose tissue was inversely associated to vigorous intensity PA among Asians[38], we did not find a lower WC for the highest intensity tertile compared to the lowest tertile. This significant lower BMI without the consistent lower WC among the Asian-Surinamese population points out that the adipose tissue might be less in other body areas except the abdominal area or they even have lower muscle mass. In order to accurately assess adiposity, its regional distribution and also lean mass, dual x-ray absorptiometry (DXA) would be a valid measuring tool [39]. Unfortunately, we were limited to the use of BMI calculations and WC measurements to define obesity and central obesity using the WHO recommended ethnic specific cut-off values (WHO 2004). However, these cut-off values have not been validated within our population.

The differences in association between body composition and PA between the Asian and African-Surinamese population could be caused by a difference in fat oxidation between ethnic groups. Compared to Caucasians, Asians have a lower level of fat oxidation at the same level of activity, whereas Africans show the same fat oxidation [20, 40]. Furthermore, compared to 
Physical activity and obesity: ethnic differences?

Caucasians, Asians need to engage in higher levels of PA for the same cardiovascular and metabolic profile $[22,41]$. Therefore, Asians might benefit from exercise at higher intensity to affect visceral adipose tissue.

Unfortunately, insufficient dietary information precluded us from taking nutrition into account. Nutrition is at least as important in weight management [42-44]. Previous studies have reported differences in dietary patterns between Asians, Africans, and Caucasians. Compared to the Caucasians, Asians had a higher energy intake, whereas Africans had similar energy intakes [45]. South Asians also have a higher intake of staple food including rice, fried rice and noodles compared to Caucasians [46]. The possible variety in diet between the Asian- and AfricanSurinamese population is definitively something which needs to be taken into consideration in future research.

Furthermore, because of the cross-sectional design, these findings are merely demonstrating associations and not necessarily prove causality. In this regard, it could be that obese participants tend to be less physically active within their leisure time and are more likely to use the car/bus for commuting to and from work, explaining the higher prevalence of obesity in individuals who were not physically active in the leisure time or commuting domain.

Finally, the combination of the four major ethnic groups, the South Asian, the Javanese, the Creole and Maroon population within respectively, the Asian- and African-Surinamese population could also be a limitation. Due to the small number of participants within each major ethnic group we decided to make these two combinations based on the region of origin. However, we acknowledge that these groups have a different cultural and genetic background that could influence the differences in association between PA characteristics and body composition. For the definition of overweight, obesity and central obesity we used BMI and WC 
cut-off values that need further validation in our population. Therefore, future studies focusing on the ethnic differences should include sufficient number of participants of each group separately.

To conclude, our results show that meeting PA recommendations, especially within the commuting and leisure time domain, are associated with reduced levels of obesity in the total population. Furthermore, in the African-Surinamese population spending more time physically active and PA within the leisure time are associated with less obesity, whereas such an association could not be observed among the Asian-Surinamese population. Our results therefore underline the targets of the WHO to increase PA in prevention of obesity [47] and emphasize the need for more in depth research into the role of PA characteristics using more objective tools in combatting the obesity epidemic in general and in different ethnic groups.

\section{Funding}

The Research Foundation Flanders (FWO) supported VC as a postdoctoral research fellow.

\section{Authors contribution}

LB and GO conceived the study and wrote the protocol. GO sought ethical approval. SB and FD analysed the data. SB, FD, VC and JT drafted the manuscript. All authors read, edited draft versions and approved the final manuscript. 


\section{References}

1. WHO: Global health risks: mortality and burden of disease attributable to selected major risks. 2009.

2. Popkin BM, Slining MM: New dynamics in global obesity facing low- and middleincome countries. Obes Rev 2013, 14 Suppl 2:11-20.

3. Di Cesare M BJ, Stevens GA, et al Trends in adult body-mass index in $\mathbf{2 0 0}$ countries from 1975 to 2014: a pooled analysis of 1698 population-based measurement studies with 19.2 million participants. Lancet 2016, 387(10026):1377-1396.

4. Bray GA, Bellanger T: Epidemiology, trends, and morbidities of obesity and the metabolic syndrome. Endocrine 2006, 29(1):109-117.

5. Sharma AM: Obesity and cardiovascular risk. Growth Horm IGF Res 2003, 13 Suppl A:S10-17.

6. Shook RP, Hand GA, Drenowatz C, Hebert JR, Paluch AE, Blundell JE, Hill JO, Katzmarzyk PT, Church TS, Blair SN: Low levels of physical activity are associated with dysregulation of energy intake and fat mass gain over 1 year. Am J Clin Nutr 2015, 102(6):1332-1338.

7. Donnelly JE, Blair SN, Jakicic JM, Manore MM, Rankin JW, Smith BK, American College of Sports M: American College of Sports Medicine Position Stand. Appropriate physical activity intervention strategies for weight loss and prevention of weight regain for adults. Medicine and science in sports and exercise 2009, 41(2):459-471.

8. Du H, Li L, Whitlock G, Bennett D, Guo Y, Bian Z, Chen J, Sherliker P, Huang Y, Zhang N et al: Patterns and socio-demographic correlates of domain-specific physical activities and their associations with adiposity in the China Kadoorie Biobank study. BMC Public Health 2014, 14:826.

9. Abu-Omar K, Rutten A: Relation of leisure time, occupational, domestic, and commuting physical activity to health indicators in Europe. Prev Med 2008, 47(3):319323. 
10. Wanner M, Gotschi T, Martin-Diener E, Kahlmeier S, Martin BW: Active transport, physical activity, and body weight in adults: a systematic review. Am J Prev Med 2012, 42(5):493-502.

11. Wanner M, Tarnutzer S, Martin BW, Braun J, Rohrmann S, Bopp M, Faeh D: Impact of different domains of physical activity on cause-specific mortality: a longitudinal study. PrevMed 2014, 62:89-95.

12. Flint E, Cummins S, Sacker A: Associations between active commuting, body fat, and body mass index: population based, cross sectional study in the United Kingdom. BMJ 2014, 349:g4887.

13. Vanhees L, Geladas N, Hansen D, Kouidi E, Niebauer J, Reiner Z, Cornelissen V, Adamopoulos S, Prescott E, Borjesson M et al: Importance of characteristics and modalities of physical activity and exercise in the management of cardiovascular health in individuals with cardiovascular risk factors: recommendations from the EACPR. Part II. EurJPrevCardiol 2012, 19(5):1005-1033.

14. Vanhees L, De SJ, Geladas N, Doyle F, Prescott E, Cornelissen V, Kouidi E, Dugmore D, Vanuzzo D, Borjesson $M$ et al: Importance of characteristics and modalities of physical activity and exercise in defining the benefits to cardiovascular health within the general population: recommendations from the EACPR (Part I). EurJPrevCardiol 2012, 19(4):670-686.

15. Dickie K, Micklesfield LK, Chantler S, Lambert EV, Goedecke JH: Cardiorespiratory Fitness and Light-Intensity Physical Activity Are Independently Associated with Reduced Cardiovascular Disease Risk in Urban Black South African Women: A CrossSectional Study. Metab Syndr Relat Disord 2016, 14(1):23-32.

16. Haskell WL, Lee IM, Pate RR, Powell KE, Blair SN, Franklin BA, Macera CA, Heath GW, Thompson PD, Bauman A: Physical activity and public health: updated recommendation for adults from the American College of Sports Medicine and the American Heart Association. Circulation 2007, 116(9):1081-1093.

17. Tillin T, Hughes AD, Mayet J, Whincup P, Sattar N, Forouhi NG, McKeigue PM, Chaturvedi $\mathrm{N}$ : The relationship between metabolic risk factors and incident cardiovascular disease 
in Europeans, South Asians, and African Caribbeans: SABRE (Southall and Brent Revisited) -- a prospective population-based study. JAmCol/Cardiol 2013, 61(17):17771786.

18. Carson AP, Howard G, Burke GL, Shea S, Levitan EB, Muntner P: Ethnic differences in hypertension incidence among middle-aged and older adults: the multi-ethnic study of atherosclerosis. Hypertension 2011, 57(6):1101-1107.

19. Gasevic D, Ross ES, Lear SA: Ethnic Differences in Cardiovascular Disease Risk Factors: A Systematic Review of North American Evidence. Can J Cardiol 2015, 31(9):1169-1179.

20. Dulloo AG, Jacquet J, Solinas G, Montani JP, Schutz Y: Body composition phenotypes in pathways to obesity and the metabolic syndrome. Int J Obes (Lond) 2010, 34 Suppl 2:S4-17.

21. White J, Jago R: Prospective associations between physical activity and obesity among adolescent girls: racial differences and implications for prevention. Arch Pediatr Adolesc Med 2012, 166(6):522-527.

22. Celis-Morales CA, Ghouri N, Bailey ME, Sattar N, Gill JM: Should physical activity recommendations be ethnicity-specific? Evidence from a cross-sectional study of South Asian and European men. PLoSOne 2013, 8(12):e82568.

23. Diemer FS, Aartman JQ, Karamat FA, Baldew SM, Jarbandhan AV, van Montfrans GA, Oehlers GP, Brewster LM: Exploring cardiovascular health: the Healthy Life in Suriname (HELISUR) study. A protocol of a cross-sectional study. BMJ open 2014, 4(12):e006380.

24. Blikman T, Stevens M, Bulstra SK, van den Akker-Scheek I, Reininga IH: Reliability and validity of the Dutch version of the International Physical Activity Questionnaire in patients after total hip arthroplasty or total knee arthroplasty. J Orthop Sports Phys Ther 2013, 43(9):650-659.

25. WHO: guidelines for data processing and analysis of the global physical activity questionnaire. 2005.

26. Consultation WHOE: Appropriate body-mass index for Asian populations and its implications for policy and intervention strategies. Lancet 2004, 363(9403):157-163. 
27. NICE: Body mass index and waist circumference thresholds for intervening to prevent ill health among black, Asian and other minority ethnic groups. The national institute for health and care excellence; 2013.

28. WHO: Waist circumference and waist-hip ratio; A report of a WHO expert consultation. Geneva: World Health Organization; 2008.

29. Coxe S, West SG, Aiken LS: The analysis of count data: a gentle introduction to poisson regression and its alternatives. J Pers Assess 2009, 91(2):121-136.

30. Ekelund U, Besson H, Luan J, May AM, Sharp SJ, Brage S, Travier N, Agudo A, Slimani N, Rinaldi $\mathrm{S}$ et al: Physical activity and gain in abdominal adiposity and body weight: prospective cohort study in 288,498 men and women. Am J Clin Nutr 2011, 93(4):826835.

31. Myers A, Gibbons C, Finlayson G, Blundell J: Associations among sedentary and active behaviours, body fat and appetite dysregulation: investigating the myth of physical inactivity and obesity. British journal of sports medicine 2016.

32. Sebastiao E, Gobbi S, Chodzko-Zajko W, Schwingel A, Papini CB, Nakamura PM, Netto AV, Kokubun E: The International Physical Activity Questionnaire-long form overestimates self-reported physical activity of Brazilian adults. Public Health 2012, 126(11):967-975.

33. Hallal PC, Gomez LF, Parra DC, Lobelo F, Mosquera J, Florindo AA, Reis RS, Pratt M, Sarmiento OL: Lessons learned after 10 years of IPAQ use in Brazil and Colombia. J Phys Act Health 2010, 7 Suppl 2:S259-264.

34. Fillipas $S$, Cicuttini $F$, Holland $A E$, Cherry $C L$ : The international physical activity questionnaire overestimates moderate and vigorous physical activity in HIV-infected individuals compared with accelerometry. J Assoc Nurses AIDS Care 2010, 21(2):173181.

35. Foong $Y C$, Aitken $D$, Winzenberg $T$, Otahal $P$, Scott $D$, Jones $G$ : The association between physical activity and reduced body fat lessens with age - results from a cross-sectional study in community-dwelling older adults. Exp Gerontol 2014, 55:107-112. 
36. Kwon S, Wang M, Hawkins M: Association between self-reported physical activity and obesity among White, Black, Hispanic, and Asian Americans: 2007 and 2009 brfss. Ethnicity \& disease 2013, 23(2):129-135.

37. Holbrook TPJSSaAL: Survey response styles across cultures. In Cross-cultural research methods in psychology. Edited by F MDvdv: Cambridge Univeristy Press; 2011:130-178.

38. Lesser IA, Yew AC, Mackey DC, Lear SA: A Cross-Sectional Analysis of the Association between Physical Activity and Visceral Adipose Tissue Accumulation in a Multiethnic Cohort. J Obes 2012, 2012:703941.

39. Toombs RJ, Ducher G, Shepherd JA, De Souza MJ: The impact of recent technological advances on the trueness and precision of DXA to assess body composition. Obesity (Silver Spring) 2012, 20(1):30-39.

40. Hall LM, Moran CN, Milne GR, Wilson J, MacFarlane NG, Forouhi NG, Hariharan N, Salt IP, Sattar N, Gill JM: Fat oxidation, fitness and skeletal muscle expression of oxidative/lipid metabolism genes in South Asians: implications for insulin resistance? PLoS One 2010, 5(12):e14197.

41. Iliodromiti S, Ghouri N, Celis-Morales CA, Sattar N, Lumsden MA, Gill JM: Should Physical Activity Recommendations for South Asian Adults Be Ethnicity-Specific? Evidence from a Cross-Sectional Study of South Asian and White European Men and Women. PLoS One 2016, 11(8):e0160024.

42. Unick JL, Jakicic JM, Marcus BH: Contribution of behavior intervention components to 24-month weight loss. Med Sci Sports Exerc 2010, 42(4):745-753.

43. Curioni CC, Lourenco PM: Long-term weight loss after diet and exercise: a systematic review. Int J Obes (Lond) 2005, 29(10):1168-1174.

44. de Munter JS, Tynelius P, Magnusson C, Rasmussen F: Longitudinal analysis of lifestyle habits in relation to body mass index, onset of overweight and obesity: results from a large population-based cohort in Sweden. Scand J Public Health 2015, 43(3):236-245.

45. Donin AS, Nightingale CM, Owen CG, Rudnicka AR, McNamara MC, Prynne CJ, Stephen AM, Cook DG, Whincup PH: Nutritional composition of the diets of South Asian, black African-Caribbean and white European children in the United Kingdom: the Child 
Heart and Health Study in England (CHASE). The British journal of nutrition 2010, 104(2):276-285.

46. Raza Q, Nicolaou M, Snijder MB, Stronks K, Seidell JC: Dietary acculturation among the South-Asian Surinamese population in the Netherlands: the HELIUS study. Public Health Nutr 2016:1-10.

47. A guide for population-based approaches to increasing levels of physical activity: implementation of the who global strategy on diet, physical activity. world health organization; 2007.

\section{Figure 1: Flowchart}

Legend: Sampling scheme of the population. Participants from other ethnicity were included in the total population analysis. 